\title{
Coorbit space theory for quasi-Banach spaces
}

\author{
by \\ Holger Rauhut (Wien)
}

\begin{abstract}
We generalize the classical coorbit space theory developed by Feichtinger and Gröchenig to quasi-Banach spaces. As a main result we provide atomic decompositions for coorbit spaces defined with respect to quasi-Banach spaces. These atomic decompositions are used to prove fast convergence rates of best $n$-term approximation schemes. We apply the abstract theory to time-frequency analysis of modulation spaces $M_{m}^{p, q}$, $0<p, q \leq \infty$.
\end{abstract}

1. Introduction. Coorbit space theory was originally developed by Feichtinger and Gröchenig $[6-8,11]$ in the late 1980's with the aim to provide a unified and group-theoretical approach to function spaces and their atomic decompositions. In particular, this theory covers the homogeneous Besov and Triebel-Lizorkin spaces and their wavelet-type atomic decompositions, as well as the modulation spaces and their Gabor-type decompositions. Recently, there has been some activity to provide generalizations to other settings than the classical one of integrable group representations $[1,9,16]$.

All the approaches taken so far cover only the case of Banach spaces. In [6] it is remarked that an extension of coorbit space theory to quasiBanach spaces would be interesting, but it seems that nothing concrete has been done since then. For instance this would allow one to describe also modulation spaces $M_{m}^{p, q}$ with $p<1$ or $q<1$, or Hardy spaces $H^{p}$ with $p<1$, as coorbit spaces. An important motivation to consider quasiBanach spaces is an application in nonlinear approximation. Indeed, the best theoretical convergence rate of best $n$-term approximations is rather small if one restricts to Banach spaces, while it can get arbitrarily large for quasi-Banach spaces.

So in this paper we extend the classical coorbit space theory to quasiBanach spaces. Our starting point is an integrable representation $\pi$ of some

2000 Mathematics Subject Classification: 42C15, 42C40, 46A16, 46E10, 46E30.

Key words and phrases: coorbit spaces, quasi-Banach spaces, Wiener amalgam spaces, modulation spaces, atomic decompositions, Gabor frames, nonlinear approximation, Lorentz spaces. 
locally compact group $\mathcal{G}$ on some Hilbert space $\mathcal{H}$. Associated to $\pi$ is the abstract wavelet transform $V_{g} f(x)=\langle f, \pi(x) g\rangle$. The crucial ingredient in coorbit space theory is the reproducing formula for $V_{g}$ (see (4.2)), which uses the group convolution on $\mathcal{G}$. Thus, it is essential to have convolution relations for certain quasi-Banach spaces $Y$ on $\mathcal{G}$. Unfortunately, even for the natural choice $Y=L^{p}(\mathcal{G}), 0<p<1$, no convolution relation is available. In order to overcome this problem we work with Wiener amalgam spaces $W\left(L^{\infty}, Y\right)$ with local component $L^{\infty}$ instead of $Y$ itself. Convolution relations for such spaces, where $Y$ is allowed to be a quasi-Banach space, were shown recently by the author in [17].

Under some technical assumption on the representation, the coorbit spaces $\mathcal{C}(Y)$ are defined as a retract of the Wiener amalgam space $W\left(L^{\infty}, Y\right)$ via the abstract wavelet transform, i.e., $\mathcal{C}(Y)=\left\{f: V_{g} f \in W\left(L^{\infty}, Y\right)\right\}$ (see Section 4). Apart from basic properties of $\mathcal{C}(Y)$ we will provide atomic decompositions of $\mathcal{C}(Y)$ of the form $\left\{\pi\left(x_{i}\right) g\right\}_{i \in I}$, where $\left(x_{i}\right)_{i \in I}$ is a suitable point set in the group (see Section 5). Based on such decompositions we will investigate approximation rates for best $n$-term approximations (Section 7 ).

Our results are applicable to time-frequency analysis on modulation spaces $M_{m}^{p, q}, 0<p, q \leq \infty$, introduced by Feichtinger [5] (see also [18, 10] for the case $p, q<1$ ). Here, we improve or give alternative proofs to some of the results of Galperin and Samarah in [10] (see Section 8).

Although in this paper we restrict $\mathcal{G}$ to be an IN group for the sake of simple presentation, we remark that nevertheless the abstract theory can be developed in general and it then also applies to homogeneous (weighted) Besov spaces $\dot{B}_{p, q}^{s}$ and Triebel-Lizorkin spaces $\dot{F}_{p, q}^{s}, 0<p, q \leq \infty$. We postpone a detailed discussion to a subsequent contribution.

2. Prerequisites. Let $\mathcal{G}$ be a locally compact group with identity $e$. For simplicity we always assume that $\mathcal{G}$ is an IN group. This means that there exists a compact neighborhood $U$ of $e$ such that $x U=U x$. IN groups are unimodular. Integration on $\mathcal{G}$ will always be with respect to the left Haar measure. We denote by $L_{x} F(y)=F\left(x^{-1} y\right)$ and $R_{x} F(y)=F(y x)$, $x, y \in \mathcal{G}$, the left and right translation operators. For a Radon measure $\mu$ we introduce the operator $\left(A_{x} \mu\right)(k)=\mu\left(R_{x} k\right), x \in \mathcal{G}$, for a continuous function $k$ with compact support. We may identify a function $F \in L^{1}$ with a measure $\mu_{F} \in M$ by $\mu_{F}(k)=\int F(x) k(x) d x$. Then clearly $A_{x} F=R_{x^{-1}} F$. Further, we define the involutions $F^{\vee}(x)=F\left(x^{-1}\right), F^{\nabla}(x)=\overline{F\left(x^{-1}\right)}$.

A quasi-norm $\|\cdot\|$ on some linear space $Y$ is defined in the same way as a norm, with the only difference that the triangle inequality is replaced by $\|f+g\| \leq C(\|f\|+\|g\|)$ with some constant $C \geq 1$. It is well-known (see e.g. [2, p. 20]) that there exists an equivalent quasi-norm $\|\cdot \mid Y\|$ on $Y$ 
and an exponent $p$ with $0<p \leq 1$ such that $\|\cdot \mid Y\|$ satisfies the $p$-triangle inequality, i.e., $\left\|f+g\left|Y\left\|^{p} \leq\right\| f\right| Y\right\|^{p}+\|g \mid Y\|^{p}$. We can choose $p=1$ if and only if $Y$ is a normed space. We always assume that such a $p$-norm on $Y$ is chosen and denote it by $\| \cdot|Y|$. If $Y$ is complete with respect to the topology defined by the metric $d(f, g)=\|f-g \mid Y\|^{p}$ then it is called a quasi-Banach space.

A quasi-Banach space of measurable functions on $\mathcal{G}$ is called solid if $F \in Y, G$ measurable and satisfying $|G(x)| \leq|F(x)|$ a.e. implies $G \in Y$ and $\|G|Y\|\leq\| F| Y\|$. The Lebesgue spaces $L^{p}(\mathcal{G}), 0<p \leq \infty$, provide natural examples of solid quasi-normed spaces on $\mathcal{G}$, and the usual quasi-norm in $L^{p}(\mathcal{G})$ is a $p$-norm if $0<p \leq 1$. If $w$ is some positive measurable weight function on $\mathcal{G}$ then we further define $L_{w}^{p}=\left\{F\right.$ measurable $\left.: F w \in L^{p}\right\}$ with $\left\|F\left|L_{w}^{p}\|:=\| F w\right| L^{p}\right\|$. A continuous weight $w$ is called submultiplicative if $w(x y) \leq w(x) w(y)$ for all $x, y \in \mathcal{G}$. Further, a weight $m$ is called $w$-moderate if $m(x y z) \leq w(x) m(y) w(z), x, y, z \in \mathcal{G}$. It is easy to see that $L_{m}^{p}$ is invariant under left and right translations if $m$ is $w$-moderate.

For a quasi-Banach space $(B, \| \cdot|B| \mid)$ we denote the quasi-norm of a bounded operator $T: B \rightarrow B$ by $\|T \mid B\|$. The symbol $A \asymp B$ indicates throughout the paper that there exist constants $C_{1}, C_{2}>0$ such that $C_{1} A \leq$ $B \leq C_{2} A$ (independent of other quantities on which $A, B$ might depend). The symbol $C$ will always denote a generic constant whose precise value might differ at different occurrences.

3. Wiener amalgam spaces. Let $B$ be one of the spaces $L^{\infty}(\mathcal{G}), L^{1}(\mathcal{G})$ or $M(\mathcal{G})$, the space of complex Radon measures. Choose some relatively compact neighborhood $Q$ of $e \in \mathcal{G}$. We define the control function by

$$
K(F, Q, B)(x):=\left\|\left(L_{x} \chi_{Q}\right) F \mid B\right\|, \quad x \in \mathcal{G},
$$

where $F$ is locally contained in $B$, in symbols $F \in B_{\text {loc }}$. Further, let $Y$ be some solid quasi-Banach space of functions on $\mathcal{G}$ containing the characteristic function of any compact subset of $\mathcal{G}$. The Wiener amalgam space $W(B, Y)$ is then defined by

$$
W(B, Y):=W(B, Y, Q):=\left\{F \in B_{\mathrm{loc}}: K(F, Q, B) \in Y\right\}
$$

with quasi-norm

$$
\|F|W(B, Y, Q)\|:=\| K(F, Q, B)| Y\| .
$$

This is indeed a $p$-norm with $p$ being the exponent of the quasi-norm of $Y$. We denote by $W\left(C_{0}, Y\right)$ the closed subspace of $W\left(L^{\infty}, Y\right)$ consisting of continuous functions. For brevity we also write

$$
\mathcal{W}(Y):=W\left(L^{\infty}, Y\right)
$$


Definition 3.1. A discrete set $X=\left(x_{i}\right)_{i \in I}$ of points in $\mathcal{G}$ is called $V$-well-spread if for relatively compact neighborhoods $V, W$ of $e$ in $\mathcal{G}$,

(a) $\mathcal{G}=\bigcup_{i \in I} x_{i} V$.

(b) For all compact sets $K \subset \mathcal{G}$ there exists a constant $C_{K}$ such that $\sup _{j \in I} \#\left\{i \in I: x_{i} K \cap x_{j} K \neq \emptyset\right\} \leq C_{K}$.

The existence of $V$-well-spread sets for arbitrarily small $V$ is proven in [4] (see also [16] for a generalization). Given a well-spread family $X=\left(x_{i}\right)_{i \in I}$, a relatively compact neighborhood $Q$ of $e \in \mathcal{G}$ and $Y$, we define the sequence space

$$
Y_{d}:=Y_{d}(X):=Y_{d}(X, Q):=\left\{\left(\lambda_{i}\right)_{i \in I}: \sum_{i \in I}\left|\lambda_{i}\right| \chi_{x_{i} Q} \in Y\right\},
$$

with natural norm $\left\|\left(\lambda_{i}\right)_{i \in I}\left|Y_{d}\|:=\| \sum_{i \in I}\right| \lambda_{i}\left|\chi_{x_{i} Q}\right| Y\right\|$. Here, $\chi_{x_{i} Q}$ denotes the characteristic function of the set $x_{i} Q$. If the quasi-norm of $Y$ is a $p$ norm, $0<p \leq 1$, then also $Y_{d}$ has a $p$-norm. For instance, if $Y=L^{p}$ then $Y_{d}=\ell^{p}(I)$.

We call a space of functions (measures) left translation invariant if all the left translations $L_{x}, x \in \mathcal{G}$, are bounded operators. Analogously, we define right translation invariance. We assume in the following that $Y$ is left and right translation invariant (although one may replace this property by a slightly more general condition). It follows from results in [17] that then also $W(B, Y)$ is left and right translation invariant and we have $\left\|L_{y}\left|W(B, Y)\|\leq\| L_{y}\right| Y\right\|,\left\|R_{y}\left|W\left(L^{\infty}, Y\right)\|\leq\| R_{y}\right| Y\right\|$ and $\left\|A_{y}\left|W(M, Y)\|\leq\| R_{y}\right| Y\right\|$. Moreover, both $W(B, Y, Q)=W(B, Y)$ and $Y_{d}=Y_{d}(X, Q)$ are complete and independent of the choice of $Q$. Since $\mathcal{G}$ is assumed to be an IN group we further have [17]

$$
\mathcal{W}\left(Y^{\vee}\right)^{\vee}=\mathcal{W}(Y) .
$$

Lemma $3.2([17])$. Let $r(x):=\left\|L_{x^{-1}} \mid \mathcal{W}(Y)\right\|$. Then $\mathcal{W}(Y)$ is continuously embedded into $L_{1 / r}^{\infty}$.

The main ingredient for the coorbit space theory with respect to quasiBanach spaces will be the following convolution relations for Wiener amalgam spaces (see [17], recalling that $\mathcal{G}$ is unimodular).

Theorem 3.3. Let $0<p \leq 1$. Let $Y$ be a p-normed right and left invariant solid quasi-Banach space. Set $w(x):=\left\|R_{x} \mid Y\right\|$. Then

$$
W(M, Y) * \mathcal{W}\left(L_{w}^{p}\right) \hookrightarrow \mathcal{W}(Y) \quad \text { and } \quad \mathcal{W}(Y) * \mathcal{W}\left(L_{w^{\vee}}^{p}\right) \hookrightarrow \mathcal{W}(Y)
$$

with corresponding estimates for the quasi-norms.

THeOREM 3.4. Let $w$ be a submultiplicative weight and $0<p \leq 1$. Then $\mathcal{W}\left(L_{w}^{p}\right) * \mathcal{W}\left(L_{w}^{p}\right) \hookrightarrow \mathcal{W}\left(L_{w}^{p}\right)$. 
Further, we will need the following maximal function. For a relatively compact neighborhood $U$ of $e \in \mathcal{G}$ and a function $G$ on $\mathcal{G}$ we define the $U$-oscillation by

$$
G_{U}^{\#}(x):=\sup _{u \in U}|G(u x)-G(x)| .
$$

The following lemma on the $U$-oscillation is an essential tool for deriving the atomic decomposition for the coorbit spaces defined later on.

LEMMA 3.5.

(a) If $G \in W\left(C_{0}, Y\right)$ then $G_{U}^{\#} \in W\left(C_{0}, Y\right)$.

(b) Let $w$ be a submultiplicative weight function and $0<p<\infty$. Then $G \in W\left(C_{0}, L_{w}^{p}\right)$ implies $\lim _{U \rightarrow\{e\}}\left\|G_{U}^{\#} \mid \mathcal{W}\left(L_{w}^{p}\right)\right\|=0$.

Proof. (a) The control function of $G_{U}^{\#}$ can be estimated as follows:

$$
\begin{aligned}
& K\left(G_{U}^{\#}, Q, L^{\infty}\right)(x)=\sup _{z \in x Q} G_{U}^{\#}(z)=\sup _{z \in x Q u \in U} \sup _{u \in U}|G(u z)-G(z)| \\
& \quad \leq \sup _{z \in x Q} \sup _{u \in U}|G(u z)|+\sup _{z \in x Q}|G(z)|=\sup _{q \in Q} \sup _{u \in U}|G(u x q)|+K\left(G, Q, C_{0}\right)(x) .
\end{aligned}
$$

Clearly, we have $K\left(G, Q, C_{0}\right) \in Y$ by assumption on $G$. We further compute the function $H(x):=\sup _{q \in Q} \sup _{u \in U}|G(u x q)|$ :

$$
\begin{aligned}
H(x) & =\sup _{q \in Q}\left\|\chi_{U}\left(R_{x q} G\right)\right\|_{\infty}=\sup _{q \in Q}\left\|\left(R_{(x q)^{-1}} \chi_{U}\right)^{\vee} G^{\vee}\right\|_{\infty} \\
& =\sup _{q \in Q}\left\|L_{(x q)^{-1}} \chi_{U^{-1}} G^{\vee}\right\|_{\infty}=\sup _{q \in Q} K\left(G^{\vee}, U^{-1}, L^{\infty}\right)^{\vee}(x q) \\
& =\left\|\chi_{x Q} K\left(G^{\vee}, U^{-1}, L^{\infty}\right)^{\vee}\right\|_{\infty}=K\left(K\left(G^{\vee}, U^{-1}, L^{\infty}\right)^{\vee}, Q, L^{\infty}\right)(x) .
\end{aligned}
$$

Thus,

$$
\begin{aligned}
\|H \mid Y\| & =\left\|K\left(K\left(G^{\vee}, U^{-1}, L^{\infty}\right)^{\vee}, Q, L^{\infty}\right) \mid Y\right\| \\
& \leq C\left\|G^{\vee} \mid \mathcal{W}\left(\mathcal{W}(Y)^{\vee}\right)\right\| .
\end{aligned}
$$

By $(3.4), \mathcal{W}\left(\mathcal{W}(Y)^{\vee}\right)^{\vee}=\mathcal{W}(\mathcal{W}(Y))$ and it is easy to see that $\mathcal{W}(\mathcal{W}(Y))=$ $\mathcal{W}(Y)$. It follows that $G_{U}^{\#} \in W\left(C_{0}, Y\right)$.

(b) By part (a), $G_{U}^{\#}$ is contained in $W\left(C_{0}, L_{w}^{p}\right)$. Let $\varepsilon>0$. Since $U \subset U_{0}$ implies $G_{U}^{\#} \leq G_{U_{0}}^{\#}$ we can find a compact set $V \subset \mathcal{G}$ such that

$$
\int_{\mathcal{G} \backslash V} K\left(G_{U}^{\#}, Q, L^{\infty}\right)(x)^{p} w(x)^{p} d x \leq \varepsilon / 2
$$

for all $U \subset U_{0}$. Since $G$ is uniformly continuous on the compact set $V Q$ we can find a neighborhood $U_{1} \subset U_{0}$ of $e$ such that

$$
G_{U_{1}}^{\#}(x) \leq M:=\frac{\varepsilon^{1 / p}}{(2|V|)^{1 / p} \nu} \quad \text { for all } x \in V Q
$$


with $\nu:=\max _{x \in V} w(x)$. This implies

$$
K\left(G_{U_{1}}^{\#}, Q, L^{\infty}\right)(x)=\sup _{z \in x Q}\left|G_{U_{1}}^{\#}(z)\right| \leq M \quad \text { for all } x \in V .
$$

Thus, we obtain

$$
\int_{V} K\left(G_{U_{1}}^{\#}, Q, L^{\infty}\right)(x)^{p} w(x)^{p} d x \leq M^{p}|V| \nu^{p}=\varepsilon / 2 .
$$

Altogether this yields $\left\|G_{U_{1}}^{\#} \mid W\left(C_{0}, L_{w}^{p}\right)\right\|^{p} \leq \varepsilon$.

4. Coorbit spaces. Let $\pi$ be an irreducible unitary representation of $\mathcal{G}$ on some Hilbert space $\mathcal{H}$. Then the abstract wavelet transform (voice transform) is defined as

$$
V_{g} f(x):=\langle f, \pi(x) g\rangle, \quad f, g \in \mathcal{H}, x \in \mathcal{G} .
$$

The representation $\pi$ is called square-integrable if there exists a non-zero $g \in \mathcal{H}$ (called admissible) such that $V_{g} g \in L^{2}(\mathcal{G})$. Since $\mathcal{G}$ is unimodular (as $\mathcal{G}$ is assumed to be an IN group) it follows from a theorem of Duflo and Moore [3] that in the case of square-integrability,

$$
\left\|V_{g} f\left|L^{2}\|=\| g\right| \mathcal{H}\right\|\|f \mid \mathcal{H}\| \quad \text { for all } f \in \mathcal{H}
$$

(provided the right normalization of the Haar measure is chosen), and actually all vectors $g \in \mathcal{H}$ are admissible.

As a consequence of (4.1), if $g$ is normalized, i.e., $\|g \mid \mathcal{H}\|=1$, we have the reproducing formula

$$
V_{g} f=V_{g} f * V_{g} g
$$

In order to introduce the coorbit spaces we first need to extend the definition of the voice transform to a larger space, the "reservoir". To this end let $v$ be some submultiplicative weight function satisfying $v \geq 1$. We define the following class of analyzing vectors:

$$
\mathbb{A}_{v}:=\left\{g \in \mathcal{H}: V_{g} g \in L_{v}^{1}\right\} .
$$

We assume that $\mathbb{A}_{v}$ is non-trivial, i.e., $\pi$ is integrable. This implies that $\pi$ is also square-integrable. With some fixed $g \in \mathbb{A}_{v} \backslash\{0\}$ we define

$$
\mathcal{H}_{v}^{1}:=\left\{f \in \mathcal{H}: V_{g} f \in L_{v}^{1}\right\}
$$

with norm $\left\|f\left|\mathcal{H}_{v}^{1}\|:=\| V_{g} f\right| L_{v}^{1}\right\|$. It can be shown [6] that $\mathcal{H}_{v}^{1}$ is a $\pi$-invariant Banach space whose definition does not depend on the choice of $g$. We denote by $\left(\mathcal{H}_{v}^{1}\right)^{\urcorner}$the anti-dual, i.e., the space of all bounded conjugate-linear functionals on $\mathcal{H}_{v}^{1}$. An equivalent norm on $\left(\mathcal{H}_{v}^{1}\right)^{\urcorner}$is given by $\left\|V_{g} f \mid L_{1 / v}^{\infty}\right\|$. Denoting by $\langle\cdot, \cdot\rangle$ also the dual pairing of $\left(\mathcal{H}_{v}^{1},\left(\mathcal{H}_{v}^{1}\right)^{\urcorner}\right)$we can extend the voice transform to $\left.\left(\mathcal{H}_{v}^{1}\right)\right\urcorner$ by

$$
V_{g} f(x)=\langle f, \pi(x) g\rangle, \quad f \in\left(\mathcal{H}_{v}^{1}\right)^{\urcorner}, g \in \mathbb{A}_{v} .
$$


Important properties of the voice transform extend to $\left.\left(\mathcal{H}_{v}^{1}\right)\right\urcorner$ as stated in the following lemma (see $[6,7]$ ).

Lemma 4.1. Let $g \in \mathbb{A}_{v}$ with $\|g \mid \mathcal{H}\|=1$.

(a) The reproducing formula extends to $\left(\mathcal{H}_{v}^{1}\right)^{\urcorner}$, i.e.,

$$
V_{g} f=V_{g} f * V_{g} g \quad \text { for all } f \in\left(\mathcal{H}_{v}^{1}\right) \text {. }
$$

(b) Conversely, if $F \in L_{1 / w}^{\infty}$ satisfies the reproducing formula $F=F * V_{g} g$ then there exists a unique element $f \in\left(\mathcal{H}_{v}^{1}\right)^{\urcorner}$such that $F=V_{g} f$.

Let us now define a space of analyzing vectors that allows us to treat also quasi-Banach spaces. For $0<p \leq 1$ and for some submultiplicative weight function $w$ we define

$$
\mathbb{B}_{w}^{p}:=\left\{g \in \mathcal{H}: V_{g} g \in \mathcal{W}\left(L_{w}^{p}\right)\right\} .
$$

In what follows, we admit only those $p$ and $w$ such that $\mathbb{B}_{w}^{p} \neq\{0\}$. Then the left and right translation invariance of $\mathcal{W}\left(L_{w}^{p}\right)$ and the irreducibility of $\pi$ imply that $\mathbb{B}_{w}^{p}$ is dense in $\mathcal{H}$. Now we are able to define the coorbit spaces.

Definition 4.2. Let $Y$ be a solid left and right translation invariant quasi-Banach space of functions on $\mathcal{G}$. Let $0<p \leq 1$ such that $Y$ has a $p$-norm and put

$$
\begin{aligned}
w(x) & :=\max \left\{\left\|R_{x}\left|Y\|,\| R_{x^{-1}}\right| Y\right\|\right\}, \\
v(x) & :=\max \left\{1,\left\|L_{x^{-1}} \mid Y\right\|\right\} .
\end{aligned}
$$

We assume that

$$
\mathbb{B}(Y):=\mathbb{B}_{w}^{p} \cap \mathbb{A}_{v}
$$

is non-trivial. Then for $g \in \mathbb{B}(Y) \backslash\{0\}$ the coorbit space is defined by

$$
\mathcal{C}(Y):=\operatorname{Co} \mathcal{W}(Y):=\left\{f \in\left(\mathcal{H}_{v}^{1}\right)^{\urcorner}: V_{g} f \in \mathcal{W}(Y)\right\}
$$

with quasi-norm $\left\|f\left|\mathcal{C}(Y)\|:=\| V_{g} f\right| \mathcal{W}(Y)\right\|$.

Let us prove that the reproducing formula extends to $\mathcal{C}(Y)$, and that $\mathcal{C}(Y)$ is complete and independent of the choice of $g \in \mathbb{B}_{w}^{p} \backslash\{0\}$.

Proposition 4.3. Let $g \in \mathbb{B}(Y)$ be such that $\|g \mid \mathcal{H}\|=1$. A function $F \in \mathcal{W}(Y)$ is of the form $V_{g} f$ for some $f \in \mathcal{C}(Y)$ if and only if $F$ satisfies the reproducing formula $F=F * V_{g} g$.

Proof. If $\left.f \in \mathcal{C}(Y) \subset\left(\mathcal{H}_{v}^{1}\right)\right\urcorner$ then $V_{g} f=V_{g} f * V_{g} g$ by the reproducing formula for $\left.\left(\mathcal{H}_{v}^{1}\right)\right\urcorner$ (see Lemma $4.1(\mathrm{a})$ ).

Conversely, assume that $F=F * V_{g} g$ for some $F \in \mathcal{W}(Y)$. By Lemma 3.2, $\mathcal{W}(Y)$ is embedded into $L_{1 / v}^{\infty}$. Thus $F \in L_{1 / v}^{\infty}$ and by Lemma 4.1(b) we have $F=V_{g} f$ for some $f \in\left(\mathcal{H}_{v}^{1}\right)^{\urcorner}$, which is then automatically contained in $\mathcal{C}(Y)$ by assumption. 
THEOREM 4.4 .

(a) $\mathcal{C}(Y)$ is a quasi-Banach space.

(b) $\mathcal{C}(Y)$ is independent of the choice of $g \in \mathbb{B}(Y) \backslash\{0\}$.

Proof. (a) Let $g \in \mathbb{B}_{w}^{p}$ such that $\|g \mid \mathcal{H}\|=1$. Assume $\left(f_{n}\right)_{n \in \mathbb{N}}$ is a Cauchy sequence in $\mathcal{C}(Y)$. This means that $V_{g} f_{n}$ is a Cauchy sequence in $\mathcal{W}(Y)$. By completeness of $\mathcal{W}(Y)$ the limit $F=\lim _{n \rightarrow \infty} V_{g} f_{n}$ in $\mathcal{W}(Y)$ exists. By Theorem 3.3 the definition of the weight $w$ implies that the operator $F \mapsto$ $F * V_{g} g$ is continuous from $\mathcal{W}(Y)$ into $\mathcal{W}(Y)$. Hence, we may interchange its application with taking limits, and together with the reproducing formula (Proposition 4.3) this yields

$$
F=\lim _{n \rightarrow \infty} V_{g} f_{n}=\lim _{n \rightarrow \infty} V_{g} f_{n} * V_{g} g=F * V_{g} g .
$$

Using Proposition 4.3 once more we see that $F=V_{g} f$ for some $f \in \mathcal{C}(Y)$. Clearly, $f=\lim _{n \rightarrow \infty} f_{n}$ in $\mathcal{C}(Y)$, and hence $\mathcal{C}(Y)$ is complete.

(b) Let $g, g^{\prime} \in \mathbb{B}_{w}^{p} \backslash\{0\}$. Without loss of generality we may assume that $g, g^{\prime}$ are normalized, i.e., $\|g\|=\left\|g^{\prime}\right\|=1$. Choose a vector $h \in \mathbb{B}_{w}^{p}$ not orthogonal to $g$ and $g^{\prime}$. It follows from the orthogonality relations that

$$
0 \neq\left\langle g^{\prime}, h\right\rangle\langle h, g\rangle V_{g} g^{\prime}=V_{g^{\prime}} g^{\prime} * V_{h} h * V_{g} g .
$$

Since $V_{g} g^{\nabla}=V_{g} g$ and likewise for $h$ and $g^{\prime}$, and since $w=w^{\vee}$, it follows from Theorem 3.4 that $V_{g} g^{\prime} \in \mathcal{W}\left(L_{w}^{p}\right)$. The inversion formula for $V_{g^{\prime}}$ reads $g=\int_{\mathcal{G}} V_{g^{\prime}} g(y) \pi(y) g^{\prime} d y$, and one easily deduces

$$
\left.V_{g} f=V_{g^{\prime}} f * V_{g} g^{\prime} \quad \text { for all } f \in\left(\mathcal{H}_{v}^{1}\right)\right\urcorner .
$$

By the convolution relation in Theorem 3.3 we conclude that $V_{g} f \in \mathcal{W}(Y)$ if $V_{g^{\prime}} f \in \mathcal{W}(Y)$. Exchanging the roles of $g$ and $g^{\prime}$ shows the converse implication.

Let us give a characterization of the space $\mathbb{B}_{w}^{p}$ of analyzing vectors.

THEOREM 4.5. Let $w$ be a submultiplicative weight and $0<p \leq 1$. Define $w^{\bullet}(x):=\max \left\{w(x), w\left(x^{-1}\right)\right\} \geq 1$. Then

$$
\mathbb{B}_{w}^{p}=\mathbb{B}_{w}^{p}=\mathcal{C}\left(L_{w}^{p} \bullet\right)
$$

Proof. Let $g \in \mathbb{B}_{w}^{p}$. It follows from $V_{g} g=V_{g} g^{\nabla}$ and (3.4) that $V_{g} g \in$ $\mathcal{W}\left(L_{w}^{p}\right)$, i.e., $g \in \mathbb{B}_{w}^{p}$. Let $g^{\prime}$ be another element of $\mathbb{B}_{w}^{p}=\mathbb{B}_{w}^{p} \bullet$. Then the previous proof shows that $V_{g} g^{\prime}, V_{g^{\prime}} g \in \mathcal{W}\left(L_{w}^{p} \bullet\right)$ and thus $g, g^{\prime} \in \mathcal{C}\left(L_{w}^{p} \bullet\right)$.

Conversely, assume that $g \in \mathcal{C}\left(L_{w}^{p} \bullet\right)$. Note that $\mathcal{C}\left(L_{w}^{p} \bullet\right) \subset \mathcal{H}$ so that voice transforms are well-defined. Let $g^{\prime} \in \mathbb{B}_{w}^{p} \backslash\{0\}$. Setting $f=g$ in (4.6) shows $V_{g} g=V_{g^{\prime}} g * V_{g} g^{\prime}=V_{g^{\prime}} g *\left(V_{g^{\prime}} g\right)^{\nabla}$. Since both $V_{g^{\prime}} g$ and $\left(V_{g^{\prime}} g\right)^{\nabla}$ are in $\mathcal{W}\left(L_{w}^{p} \bullet\right.$ by $(3.4)$, it follows from Theorem 3.4 that $V_{g} g \in \mathcal{W}\left(L_{w}^{p} \bullet\right.$, i.e., $g \in \mathbb{B}_{w}^{p} \bullet$. 
The following theorem will be useful to prove a weak version of a conjecture in [10, Conjecture 12].

THEOREM 4.6. Let $w$ be a submultiplicative weight function satisfying $w=w^{\vee}$ and assume $0<p \leq 1$. If $V_{g} f \in \mathcal{W}\left(L_{w}^{p}\right)$ for $f, g \in \mathcal{H}$ then both $f$ and $g$ are in $\mathbb{B}_{w}^{p}=\mathcal{C}\left(L_{w}^{p}\right)$.

Proof. It follows from (4.6) that

$$
V_{g} g=\left(V_{g} f\right)^{\nabla} * V_{g} f \quad \text { and } \quad V_{f} f=V_{g} f *\left(V_{g} f\right)^{\nabla} .
$$

Since $w(x)=w\left(x^{-1}\right)$ we conclude by (3.4) that also $\left(V_{g} f\right)^{\nabla}$ lies in $\mathcal{W}\left(L_{w}^{p}\right)$. The convolution relation in Theorem 3.4 and application of Theorem 4.5 finally yield the assertion.

5. Discretizations. Our next result is concerned with atomic decompositions for coorbit spaces.

TheOREM 5.1. Let $g \in \mathbb{B}(Y) \backslash\{0\}$. Then there exists a compact neighborhood $U$ of e such that for any $U$-dense well-spread set $X=\left(x_{i}\right)_{i \in I}$ the family $\left\{\pi\left(x_{i}\right) g\right\}_{i \in I}$ forms an atomic decomposition of $\mathcal{C}(Y)$. This means that there exists a sequence $\left(\lambda_{i}\right)_{i \in I}$ of bounded linear functionals on $\left(\mathcal{H}_{v}^{1}\right)^{\urcorner}$ (not necessarily unique) such that

(a) $f=\sum_{i \in I} \lambda_{i}(f) \pi\left(x_{i}\right) g$ for all $f \in \mathcal{C}(Y)$ with convergence in the weak* topology of $\left.\left(\mathcal{H}_{v}^{1}\right)\right\urcorner$, and in the quasi-norm topology of $\mathcal{C}(Y)$ provided the finite sequences are dense in $Y_{d}$;

(b) an element $f \in\left(\mathcal{H}_{v}^{1}\right)^{\urcorner}$is in $\mathcal{C}(Y)$ if and only if $\left(\lambda_{i}(f)\right)_{i \in I} \in Y_{d}$ and

$$
\left\|\left(\lambda_{i}(f)\right)_{i \in I}\left|Y_{d}\|\asymp\| f\right| \mathcal{C}(Y)\right\| \quad \text { for all } f \in \mathcal{C}(Y) .
$$

Proof. The theorem is proven analogously to the Banach space case (see $[6$, Sections 5, 6] or [16]). We do not give the details but rather note that the basic ingredient is a discretization of the reproducing formula (4.2). Instead of convolution relations for $Y$ as in [6], the corresponding relations for $\mathcal{W}(Y)$ and $W(M, Y)$ stated in Theorems 3.3 and 3.4 are heavily used. Also Lemma 3.5 is needed, and the usual triangle inequality has to be replaced by the $p$-triangle inequality.

In certain situations one might be able to construct frame expansions as in (5.1) below on the level of the Hilbert space $\mathcal{H}$. The next theorem states that such expansions extend automatically from $\mathcal{H}$ to general coorbit spaces under certain assumptions. Its proof is a modification of the one in [13] again replacing convolution relations for $Y$ by the corresponding ones for $\mathcal{W}(Y)$ and the usual triangle inequality by the $p$-triangle inequality. 
Theorem 5.2. Let $g_{r}, \gamma_{r} \in \mathbb{B}(Y), r=1, \ldots, n$, and $X=\left(x_{i}\right)_{i \in I}$ be $a$ well-spread set such that

$$
f=\sum_{r=1}^{n} \sum_{i \in I}\left\langle f, \pi\left(x_{i}\right) \gamma_{r}\right\rangle \pi\left(x_{i}\right) g_{r} \quad \text { for all } f \in \mathcal{H} .
$$

Then expansion (5.1) extends to all $f \in \mathcal{C}(Y)$ with norm convergence if the finite sequences are dense in $Y_{d}$ and with weak ${ }^{*}$ convergence in general. Moreover, $\left.f \in\left(\mathcal{H}_{v}^{1}\right)\right\urcorner$ is in $\mathcal{C}(Y)$ if and only if $\left(\left\langle f, \pi\left(x_{i}\right) \gamma_{r}\right\rangle\right)_{i \in I}$ lies in $Y_{d}$ for each $r=1, \ldots, n$, and

$$
\left\|\left(\left(\left\langle f, \pi\left(x_{i}\right) \gamma_{r}\right\rangle\right)_{i \in I}\right)_{r=1}^{n}\left|\bigoplus_{r=1}^{n} Y_{d}\|\asymp\| f\right| \mathcal{C}(Y)\right\| \quad \text { for all } f \in \mathcal{C}(Y) .
$$

6. Characterizations of $\mathcal{C}(Y)$ via $Y$. The original definition of the coorbit spaces by Feichtinger and Gröchenig involves $Y$ rather than $\mathcal{W}(Y)$. It is interesting to investigate what happens if we replace $\mathcal{W}(Y)$ by $Y$ in our more general case. In order to distinguish clearly between the two spaces let us write $\left.\operatorname{Co} Y=\left\{f \in\left(\mathcal{H}_{v}^{1}\right)\right\urcorner: V_{g} f \in Y\right\}$ with natural norm $\|f \mid \operatorname{Co} Y\|=$ $\left\|V_{g} f \mid Y\right\|$, and $\operatorname{Co} \mathcal{W}(Y)=\mathcal{C}(Y)$ as usual. It was already proven in [8] that in the classical Banach space case both spaces coincide:

Theorem 6.1 (Theorem 8.3 in [8]). Let $Y$ be a solid Banach space of functions on $\mathcal{G}$ that is left and right translation invariant and continuously embedded into $L_{\text {loc }}^{1}(\mathcal{G})$. Then $\operatorname{Co} Y=\operatorname{Co} \mathcal{W}(Y)$ with equivalent norms.

In the general case of quasi-Banach spaces at least the inclusion $\operatorname{Co} \mathcal{W}(Y)$ $\subset$ Co $Y$ holds since $\mathcal{W}(Y) \subset Y$. However, it seems doubtful that we can state results on the converse inclusion in the general abstract case. Moreover, it is even not clear whether Co $Y$ is a complete space.

In special cases, however, one might be able to prove that $\left\|V_{g} f \mid \mathcal{W}(Y)\right\|$ $\leq C\left\|V_{g} f \mid Y\right\|$ for a very specific choice of $g$, by using methods that are not available in the abstract setting (like analyticity properties for instance): see e.g. Section 8. Then one may extend this inequality to more general analyzing vectors $g$ as shown by the next result.

THEOREM 6.2. Let $Y$ be a left and right translation invariant solid $p$ normed quasi-Banach space and let $v$ be the function defined in (4.4). Assume that there exists a non-zero vector $g_{0} \in \mathbb{B}(Y)$ and a constant $C>0$ such that $V_{g_{0}} f \in Y$ if $V_{g_{0}} f \in \mathcal{W}(Y)$ and

$$
\left\|V_{g_{0}} f\left|\mathcal{W}(Y)\|\leq C\| V_{g_{0}} f\right| Y\right\|
$$

for all $f \in\left(\mathcal{H}_{v}^{1}\right)^{\urcorner}$. Let $g \in \mathbb{B}(Y) \backslash\{0\}$ be arbitrary. Then

$$
\left\|V_{g} f\left|\mathcal{W}(Y)\|\asymp\| V_{g} f\right| Y\right\|
$$

for all $\left.f \in\left(\mathcal{H}_{v}^{1}\right)\right\urcorner$ and $\left.\operatorname{Co} \mathcal{W}(Y)=\operatorname{Co} Y=\left\{f \in\left(\mathcal{H}_{v}^{1}\right)\right\urcorner, V_{g} f \in Y\right\}$. In particular, Co $Y$ is complete. 
Proof. Since $\mathcal{C}(Y)$ is independent of the choice of $g \in \mathbb{B}(Y) \backslash\{0\}$ (Theorem 4.4) we have $\left\|V_{g} f\left|\mathcal{W}(Y)\|\leq C\| V_{g_{0}} f\right| \mathcal{W}(Y)\right\|$ for all $f \in\left(\mathcal{H}_{v}^{1}\right)^{\urcorner}$. Thus, it remains to prove that $\left\|V_{g_{0}} f\left|Y\|\leq C\| V_{g} f\right| Y\right\|$ for all $\left.f \in\left(\mathcal{H}_{v}^{1}\right)\right\urcorner$. By the assumptions on $g$ it follows from Theorem 5.1 that $g_{0}$ has a decomposition

$$
g_{0}=\sum_{i \in I} \lambda_{i}\left(g_{0}\right) \pi\left(x_{i}\right) g
$$

with $\left(\lambda_{i}\left(g_{0}\right)\right)_{i \in I} \in \ell_{w}^{p}=\left(L_{w}^{p}\right)_{d}$ and $\left\|\left(\lambda_{i}\left(g_{0}\right)\right)_{i \in I}\left|\ell_{w}^{p}\|\asymp\| g_{0}\right| \mathcal{C}\left(L_{w}^{p}\right)\right\|$, where $w$ is the weight in (4.3). Hence, we obtain

$$
V_{g_{0}} f(x)=\left\langle f, \pi(x) g_{0}\right\rangle=\left\langle f, \pi(x) \sum_{i \in I} \lambda_{i}\left(g_{0}\right) \pi\left(x_{i}\right) g\right\rangle=\sum_{i \in I} \overline{\lambda_{i}\left(g_{0}\right)} R_{x_{i}} V_{g} f(x) .
$$

By the $p$-triangle inequality this yields

$$
\begin{aligned}
\left\|V_{g_{0}} f \mid Y\right\|^{p} & =\left\|\sum_{i \in I} \overline{\lambda_{i}\left(g_{0}\right)} R_{x_{i}} V_{g} f\left|Y\left\|^{p} \leq \sum_{i \in I}\left|\lambda_{i}\left(g_{0}\right)\right|^{p}\right\| R_{x_{i}}\right| Y\right\|^{p}\left\|V_{g} f \mid Y\right\|^{p} \\
& \leq C\left\|g_{0}\left|\mathcal{C}\left(L_{v}^{p}\right)\left\|^{p}\right\| V_{g} f\right| Y\right\|^{p}
\end{aligned}
$$

for all $\left.f \in\left(\mathcal{H}_{v}^{1}\right)\right\urcorner$. The reverse inequality $\left\|V_{g} f\left|Y\|\leq\| V_{g} f\right| \mathcal{W}(Y)\right\|$ is clear.

7. Nonlinear approximation. Let us now discuss nonlinear approximation. Let $\left(x_{i}\right)_{i \in I}$ be some well-spread set and $g$ such that $\left\{\pi\left(x_{i}\right) g\right\}_{i \in I}$ forms an atomic decomposition of the coorbit space we want to consider. We denote by

$$
\sigma_{n}(f, \mathcal{C}(Y)):=\inf _{N \subset I, \# N \leq n}\left\|f-\sum_{i \in N} \lambda_{i} \pi\left(x_{i}\right) g \mid \mathcal{C}(Y)\right\|
$$

the error of best $n$-term approximation in $\mathcal{C}(Y)$. Here, the infimum is also taken over all possible choices of coefficients $\lambda_{i}$. Our task is to find a class of elements for which this error has a certain decay when $n$ tends to $\infty$.

To this end we consider coorbit spaces with respect to Lorentz spaces $L(p, \infty)$, also called weak $L^{p}$ spaces. For some measurable function $F$ on $\mathcal{G}$ let $\lambda_{F}(s)=|\{x:|F(x)|>s\}|$ be its distribution function, where $|\cdot|$ denotes the Haar measure of a set. Then the nonincreasing rearrangement of $F$ is defined as $F^{*}(t)=\inf \left\{s: \lambda_{F}(s) \leq t\right\}$. We let

$$
\|F\|_{p, \infty}^{*}:=\sup _{t>0} t^{1 / p} F^{*}(t) .
$$

The Lorentz space $L(p, \infty)$ is defined as the collection of all $F$ such that the quantity above is finite. If $p>1$ then $L(p, \infty)$ is a Banach space, and for $p \leq 1$ it is a quasi-Banach space. Moreover, in the latter case there exists an equivalent $r$-norm for any $r<p$. We note also that $L^{p} \subset L(p, \infty)$. For more information on Lorentz spaces we refer e.g. to [15].

By the properties of the Haar measure it is easily seen that all spaces $L(p, \infty)$ are left and right translation invariant. Thus, if $m$ is a moder- 
ate function then also $L_{m}(p, \infty)=\{F$ measurable : $F m \in L(p, \infty)\}$ with the quasi-norm $\left\|F \mid L_{m}(p, \infty)\right\|:=\|F m\|_{p, \infty}^{*}$ is left and right translation invariant. In particular, the Wiener amalgam spaces $\mathcal{W}\left(L_{m}(p, \infty)\right)$ are welldefined. Further, if $\mathbb{B}\left(L_{m}(p, \infty)\right)$ (see (4.5)) is nontrivial then also the coorbit space $\mathcal{C}\left(L_{m}(p, \infty)\right)$ is well-defined.

It is not difficult to see that the sequence space $\left(L_{m}(p, \infty)\right)_{d}(X)$ associated to a well-spread set $X=\left(x_{i}\right)_{i \in I}$ coincides with a Lorentz space $\ell_{m}(p, \infty)$ on the index set $I$. In particular, an equivalent quasi-norm on $\left(L_{m}(p, \infty)\right)_{d}(X)$ is given by

$$
\left\|\left(\lambda_{i}\right)_{i \in I} \mid \ell_{m}(p, \infty)\right\|=\sup _{n \in \mathbb{N}} n^{1 / p}(\lambda m)^{*}(n)
$$

where $(\lambda m)^{*}$ denotes the nonincreasing rearrangement of the sequence $\left(\lambda_{i} m\left(x_{i}\right)\right)_{i \in I}$.

THEOREM 7.1. Let $m$ be some $w$-moderate weight function on $\mathcal{G}$, let $0<p<q \leq \infty$ and define $\alpha=1 / p-1 / q>0$. Let $\left(x_{i}\right)_{i \in I}$ be a wellspread set and $g \in \mathbb{B}\left(L_{m}(p, \infty)\right) \subset \mathbb{B}\left(L_{m}^{q}\right)$ such that $\left\{\pi\left(x_{i}\right) g\right\}_{i \in I}$ forms an atomic decomposition simultaneously of $\mathcal{C}\left(L_{m}(p, \infty)\right)$ and $\mathcal{C}\left(L_{m}^{q}\right)$ (according to Theorem 5.1). Then for all $f \in \mathcal{C}\left(L_{m}(p, \infty)\right)$,

$$
\sigma_{n}\left(f, \mathcal{C}\left(L_{m}^{q}\right)\right) \leq C\left\|f \mid \mathcal{C}\left(L_{m}(p, \infty)\right)\right\| n^{-\alpha} .
$$

Proof. Let $f=\sum_{i \in I} \lambda_{i}(f) \pi\left(x_{i}\right) g$ be an expansion of $f \in \mathcal{C}\left(L_{m}(p, \infty)\right)$ in terms of the atomic decomposition. By Theorem 5.1 we have $(\lambda m)_{k}^{*} \leq$ $C\left\|f \mid \mathcal{C}\left(L_{m}(p, \infty)\right)\right\| k^{-1 / p}$. Let $\tau: \mathbb{N} \rightarrow I$ be a bijection that realizes the nonincreasing rearrangement, i.e., $\lambda_{\tau(k)} m\left(x_{\tau(k)}\right)=(\lambda m)_{k}^{*}$. Moreover, $\left(L_{m}^{q}\right)_{d}=$ $\ell_{m}^{q}(I)$, and $\left\|\left(\lambda_{i}(f)\right)_{i \in I} \mid \ell_{m}^{q}(I)\right\|$ forms an equivalent norm on $\mathcal{C}\left(L_{v}^{q}\right)$ once again by Theorem 5.1. We obtain

$$
\begin{aligned}
& \sigma_{n}\left(f, \mathcal{C}\left(L_{m}^{q}\right)\right) \leq\left\|f-\sum_{k=1}^{n} \lambda_{\tau(k)} \pi\left(x_{\tau(k)}\right) g \mid \mathcal{C}\left(L_{m}^{q}\right)\right\| \\
& =\left\|\sum_{k=n+1}^{\infty} \lambda_{\tau(k)} \pi\left(x_{\tau(k)}\right) g \mid \mathcal{C}\left(L_{m}^{q}\right)\right\| \leq C\left(\sum_{k=n+1}^{\infty}\left((\lambda m)_{k}^{*}\right)^{q}\right)^{1 / q} \\
& \leq C\left\|f\left|\mathcal{C}\left(L_{m}(p, \infty)\right)\left\|\left(\sum_{k=n+1}^{\infty} k^{-q / p}\right)^{1 / q} \leq C\right\| f\right| \mathcal{C}\left(L_{m}(p, \infty)\right)\right\| n^{-\alpha} .
\end{aligned}
$$

This completes the proof.

REMARK 7.2.

(a) The obvious embedding $\mathcal{C}\left(L_{m}^{p}\right) \subset \mathcal{C}\left(L_{m}(p, \infty)\right)$ implies that we also have $\sigma_{n}\left(f, \mathcal{C}\left(L_{m}^{q}\right)\right) \leq C n^{1 / q-1 / p}$ for all $f \in \mathcal{C}\left(L_{m}^{p}\right)$ if $p<q$.

(b) In order to have a very fast decay of $\sigma_{n}\left(f, \mathcal{C}\left(L_{m}^{q}\right)\right)$ one obviously has to take $p$ very small in the theorem above, in particular, $p \leq 1$. 
Clearly, $\mathcal{C}\left(L_{m}(p, \infty)\right)$ is no longer a Banach space in this case, but only a quasi-Banach space. So it is very natural to treat also the case of quasi-Banach spaces when dealing with problems in nonlinear approximation. This was actually one of the motivations for this paper.

8. Modulation spaces. Let $\mathbb{H}_{d}:=\mathbb{R}^{d} \times \mathbb{R}^{d} \times \mathbb{T}$ denote the (reduced) Heisenberg group with group law $(x, \omega, \tau)\left(x^{\prime}, \omega^{\prime}, \tau^{\prime}\right)=\left(x+x^{\prime}, \omega+\omega^{\prime}\right.$, $\left.\tau \tau^{\prime} e^{\pi i\left(x^{\prime} \cdot \omega-x \cdot \omega^{\prime}\right)}\right)$. It is an IN group and thus unimodular. Its Haar measure is given by

$$
\int_{\mathbb{H}_{d}} f(h) d h=\iint_{\mathbb{R}^{d}} \int_{\mathbb{R}^{d}}^{1} f\left(x, \omega, e^{2 \pi i t}\right) d t d \omega d x .
$$

We denote by $T_{x} f(t):=f(t-x)$ and $M_{\omega} f(t)=e^{2 \pi i \omega \cdot t} f(t), x, \omega, t \in \mathbb{R}^{d}$, the translation and modulation operators on $L^{2}\left(\mathbb{R}^{d}\right)$. Then the Schrödinger representation $\varrho$ is defined by

$$
\varrho(x, \omega, \tau):=\tau e^{\pi i x \cdot \omega} T_{x} M_{\omega}=\tau e^{-\pi i x \cdot \omega} M_{\omega} T_{x} .
$$

It is well-known that this is an irreducible unitary and square-integrable representation of $\mathbb{H}_{d}$. The corresponding voice transform is essentially the short time Fourier transform:

$$
\begin{aligned}
V_{g} f & (x, \omega, \tau) \\
& =\langle f, \varrho(x, \omega, \tau) g\rangle_{L^{2}\left(\mathbb{R}^{d}\right)}=\bar{\tau} \int_{\mathbb{R}^{d}} f(t) \overline{e^{-\pi i x \cdot \omega} M_{\omega} T_{x} g(t)} d t \\
& =\bar{\tau} e^{\pi i x \cdot \omega} \int_{\mathbb{R}^{d}} f(t) \overline{g(t-x)} e^{-2 \pi i t \cdot \omega} d t=\bar{\tau} e^{\pi i x \cdot \omega} \operatorname{STFT}_{g} f(x, \omega) .
\end{aligned}
$$

Let us now introduce the modulation spaces on $\mathbb{R}^{d}$. We consider nonnegative continuous weight functions $m$ on $\mathbb{R}^{d} \times \mathbb{R}^{d}$ that satisfy

$$
m(x+y, \omega+\xi) \leq C\left(1+|x|^{2}+|\omega|^{2}\right)^{a / 2} m(y, \xi), \quad(x, \omega),(y, \xi) \in \mathbb{R}^{d} \times \mathbb{R}^{d},
$$

for some constants $C>0, a \geq 0$. This means that $m$ is a $w$-moderate function for $w(x, \omega)=\left(1+|x|^{2}+|\omega|^{2}\right)^{a / 2}$ (see also [12, Chapter 11.1]). Additionally, we require $m$ to be symmetric, i.e., $m(-x,-\omega)=m(x, \omega)$. A typical choice is $m_{s}(x, \omega)=(1+|\omega|)^{s}, s \in \mathbb{R}$. For $0<p, q \leq \infty$ and $m$ as above we introduce $L_{m}^{p, q}:=L_{m}^{p, q}\left(\mathbb{R}^{2 d}\right):=\left\{F\right.$ measurable $\left.:\left\|F \mid L_{m}^{p, q}\right\|<\infty\right\}$ with quasi-norm

$$
\left\|F \mid L_{m}^{p, q}\right\|:=\left(\int_{\mathbb{R}^{d}}\left(\int_{\mathbb{R}^{d}}|F(x, \omega)|^{p} m(x, \omega)^{p} d x\right)^{q / p} d \omega\right)^{1 / q} .
$$

This expression is an $r$-norm with $r:=\min \{1, p, q\}$. 
Let $g$ be some nonzero Schwartz function on $\mathbb{R}^{d}$. The short time Fourier transform $\mathrm{STFT}_{g}$ extends to the space $\mathcal{S}^{\prime}\left(\mathbb{R}^{d}\right)$ of tempered distributions in a natural way. Given $0<p, q \leq \infty$ and $m$ as above, the modulation space is defined as

$$
M_{m}^{p, q}:=\left\{f \in \mathcal{S}^{\prime}\left(\mathbb{R}^{d}\right):\left\|\operatorname{STFT}_{g} f \mid L_{m}^{p, q}\right\|<\infty\right\}
$$

with quasi-norm $\left\|f\left|M_{m}^{p, q}\|=\| \operatorname{STFT}_{g} f\right| L_{m}^{p, q}\right\|$. Since by (8.1), $\left|V_{g} f(x, \omega, \tau)\right|$ $=\left|\mathrm{STFT}_{g} f(x, \omega)\right|$, we can identify the modulation spaces with coorbit spaces,

$$
M_{m}^{p, q}\left(\mathbb{R}^{d}\right)=\operatorname{Co} L_{m}^{p, q}\left(\mathbb{H}_{d}\right)=\left\{f \in \mathcal{S}^{\prime}: V_{g} f \in L_{m}^{p, q}\right\},
$$

where $m$ and $L_{m}^{p, q}$ are extended to $\mathbb{H}_{d}$ in an obvious way, e.g. $m(x, \omega, \tau)=$ $m(x, \omega)$. However, at the moment we do not know yet whether Co $L_{m}^{p, q}$ coincides with

$$
\mathcal{C}\left(L_{m}^{p, q}\right)=\left\{f \in \mathcal{S}^{\prime}\left(\mathbb{R}^{d}\right): V_{g} f \in \mathcal{W}\left(L_{m}^{p, q}\right)\right\},
$$

if $p<1$ or $q<1$. It is not even clear yet whether $M_{m}^{p, q}$ is complete. We will use Theorem 6.2 and a result from [10] to clarify this problem. Let us first investigate the space $\mathbb{B}\left(L_{m}^{p, q}\right)$ (see Definition 4.2). It is indeed not hard to see that $\mathbb{B}_{w}^{r} \subset \mathbb{B}\left(L_{m}^{p, q}\right)$ with $r=\min \{1, p, q\}$, and Theorem 4.5 yields

$$
\mathbb{B}_{w}^{r}=\mathcal{C}\left(L_{w}^{r}\right)
$$

Let $g_{0}(t)=e^{-\pi|t|^{2}}$ be a Gaussian. Using the relation of $\operatorname{STFT}_{g_{0}}$ to the Bargmann transform, Galperin and Samarah proved that

$$
\left\|V_{g_{0}} f\left|\mathcal{W}\left(L_{m}^{p, q}\right)\|\leq C\| V_{g_{0}} f\right| L_{m}^{p, q}\right\|
$$

for all $f \in M_{m}^{p, q}[10$, Lemma 3.2]. Thus, it follows from Theorem 6.2 that

$$
\mathcal{C}\left(L_{m}^{p, q}\right)=M_{m}^{p, q},
$$

and the latter is complete. It seems that the completeness of $M_{m}^{p, q}$ for $p<1$ or $q<1$ was not stated in [10] or elsewhere in the literature although its proof is somehow hidden in [10].

The abstract discretization Theorem 5.1 yields the following result for Gabor type atomic decompositions of modulation spaces.

THEOREM 8.1. Let $0<p_{0} \leq 1$ and $w$ be some symmetric submultiplicative weight function on $\mathbb{R}^{d} \times \mathbb{R}^{\bar{d}}$ with polynomial growth. Let $g \in M_{w}^{p_{0}}$. Then there exist constants $a, b>0$ such that

$$
\left\{M_{b j} T_{a k} g: k, j \in \mathbb{Z}^{d}\right\}
$$

forms an atomic decomposition for all modulation spaces $M_{m}^{p, q}$ with $p_{0} \leq$ $p, q \leq \infty$ and $m$ being a w-moderate weight. This means that there exist 
functionals $\lambda_{k, j}, k, j \in \mathbb{Z}^{d}$, on $M_{1 / w}^{\infty}\left(\subset \mathcal{S}^{\prime}\right)$ such that

(a) any $f \in M_{m}^{p, q}$ has the series expansion $f=\sum_{k, j \in \mathbb{Z}^{d}} \lambda_{k, j}(f) M_{b j} T_{a k} g$;

(b) a distribution $f \in M_{1 / w}^{\infty}$ belongs to $M_{m}^{p, q}$ if and only if $\left(\lambda_{k, j}(f)\right)_{k, j \in \mathbb{Z}^{d}}$ belongs to $\ell_{m}^{p, q}\left(\mathbb{Z}^{2 d}\right)$, and we have the quasi-norm equivalence

$$
\begin{aligned}
\left\|f \mid M_{m}^{p, q}\right\| & \asymp\left(\sum_{j \in \mathbb{Z}^{d}}\left(\sum_{k \in \mathbb{Z}^{d}}\left|\lambda_{k, j}(f)\right|^{p} m(a k, b j)^{p}\right)^{q / p}\right)^{1 / q} \\
& =:\left\|\left(\lambda_{k, j}(f)\right) \mid \ell_{m}^{p, q}\left(\mathbb{Z}^{2 d}\right)\right\| .
\end{aligned}
$$

We remark that the abstract Theorem 5.1 allows extending the previous result also to irregular Gabor frames on $M_{m}^{p, q}$.

Theorem 8.1 indicates that the modulation spaces $M_{w}^{p_{0}}$ with $0<p_{0} \leq 1$ are the correct window classes for time-frequency analysis on $M_{m}^{p, q}$. This was already conjectured in [10]. Galperin and Samarah also conjectured that whenever $V_{g} f \in L_{v}^{p}$ then $f \in M_{v}^{p}$ and $g \in M_{v}^{p}$ [10, Conjecture 12]. Theorem 4.6 leads to a weak version of this conjecture.

THEOREM 8.2. Let $f, g \in L^{2}\left(\mathbb{R}^{d}\right)$ and $0<p \leq 1$. Let $v$ be a symmetric submultiplicative weight function. If $V_{g} f \in \mathcal{W}\left(L_{v}^{p}\right)$, then $g \in M_{v}^{p}$ and $f \in M_{v}^{p}$.

The remaining question is whether $V_{g} f \in L_{v}^{p}$ already implies that $V_{g} f \in \mathcal{W}\left(L_{v}^{p}\right)$

Let us also apply Theorem 5.2 to our situation.

THEOREM 8.3. Let $g \in \mathcal{S}\left(\mathbb{R}^{d}\right)$ and $a, b>0$ be such that

$$
\left\{M_{b j} T_{a k} g: j, k \in \mathbb{Z}^{d}\right\}
$$

forms a Gabor frame for $L^{2}\left(\mathbb{R}^{d}\right)$. Then its canonical dual $\gamma$ is also contained in $\mathcal{S}\left(\mathbb{R}^{d}\right)$, and any $f \in M_{m}^{p, q}, 0<p, q \leq \infty$, has a decomposition

$$
f=\sum_{j, k \in \mathbb{Z}^{d}}\left\langle f, M_{b j} T_{a k} g\right\rangle M_{b j} T_{a k} \gamma
$$

with $\left\|f\left|M_{m}^{p, q}\|\asymp\|\left(\left\langle f, M_{b j} T_{a k} g\right\rangle\right)_{j, k \in \mathbb{Z}^{d}}\right| \ell_{m}^{p, q}\left(\mathbb{Z}^{2 d}\right)\right\|$.

Proof. Since (8.2) forms a Gabor frame with dual window $\gamma$, any $f$ in $L^{2}\left(\mathbb{R}^{d}\right)$ has a decomposition

$$
f=\sum_{j, k \in \mathbb{Z}^{d}}\left\langle f, M_{b j} T_{a k} g\right\rangle M_{b j} T_{a k} \gamma .
$$

It was shown in [12, Corollary 13.5.4] that also the dual window $\gamma$ is contained in $\mathcal{S}\left(\mathbb{R}^{d}\right)$. Since $\mathcal{S}\left(\mathbb{R}^{d}\right) \subset M_{m}^{p, q}$ for all $0<p, q \leq \infty$ and all $w$-moderate weights $m$ with $w$ having polynomial growth, we have $g, \gamma \in \mathbb{B}\left(L_{m}^{p, q}\right)=M_{w}^{r}$ with $r=\min \{1, p, q\}$. Clearly, the set $\left\{(a k, b j): k, j \in \mathbb{Z}^{d}\right\}$ is well-spread in $\mathbb{H}^{d}$. Thus, the assertion follows from Theorem 5.2. 
Of course, one can also apply Theorem 7.1 to best $n$-term approximations with Gabor frames; cf. also [14] for approximation with local Fourier bases. We leave this straightforward task to the interested reader.

Acknowledgements. Major parts of this paper were developed during a stay at the Mathematical Institute of the University of Wrocław, Poland. The author would like to thank its members for their warm hospitality. In particular, he expresses his gratitude to M. Paluszyński and R. Szwarc for interesting discussions on the subject. The author was supported by the European Union's Human Potential Programme under contracts HPRNCT-2001-00273 (HARP) and HPRN-CT-2002-00285 (HASSIP).

\section{References}

[1] S. Dahlke, G. Steidl, and G. Teschke, Coorbit spaces and Banach frames on homogeneous spaces with applications to the sphere, Adv. Comp. Math. 21 (2004), $147-180$.

[2] R. DeVore and G. G. Lorentz, Constructive Approximation, Springer, New York, 1993.

[3] M. Duflo and C. C. Moore, On the regular representation of a nonunimodular locally compact group, J. Funct. Anal. 21 (1976), 209-243.

[4] H. G. Feichtinger, A characterization of minimal homogeneous Banach spaces, Proc. Amer. Math. Soc. 81 (1981), 55-61.

[5] - Modulation spaces on locally compact Abelian groups, in: R. Radha et al. (eds.), Proc. Internat. Conf. on Wavelets and Applications (Chennai, 2002), New Delhi Allied Publ., 2003, 1-56.

[6] H. G. Feichtinger and K. Gröchenig, A unified approach to atomic decompositions via integrable group representations, in: Functions, Spaces and Applications (Lund, 1986), Lecture Notes in Math. 1302, Springer, 1988, 52-73.

[7] - - - Banach spaces related to integrable group representations and their atomic decompositions I, J. Funct. Anal. 86 (1988), 307-340.

[8] - - - Banach spaces related to integrable group representations and their atomic decompositions II, Monatsh. Math. 108 (1989), 129-148.

[9] M. Fornasier and H. Rauhut, Continuous frames, function spaces, and the discretization problem, J. Fourier Anal. Appl. 11 (2005), 245-287.

[10] Y. V. Galperin and S. Samarah, Time-frequency analysis on modulation spaces $M_{m}^{p, q}, 0<p, q \leq \infty$, Appl. Comput. Harm. Anal. 16 (2004), 1-18.

[11] K. Gröchenig, Describing functions: atomic decompositions versus frames, Monatsh. Math. 112 (1991), 1-41.

[12] - Foundations of Time-Frequency Analysis, Birkhäuser, 2001.

[13] - Unconditional bases in translation and dilation invariant function spaces on $\mathbb{R}^{n}$, in: B. Sendov et al. (eds.), Constructive Theory of Functions (Varna, 1987), 1988, 174-183.

[14] K. Gröchenig and S. Samarah, Nonlinear approximation with local Fourier bases, Constr. Approx. 16 (2000), 317-331.

[15] R. A. Hunt, On L(p,q) spaces, Enseign. Math. 12 (1966), 249-276.

[16] H. Rauhut, Banach frames in coorbit spaces consisting of elements which are invariant under symmetry groups, Appl. Comput. Harm. Anal. 18 (2005), 94-122. 
[17] H. Rauhut, Wiener amalgam spaces with respect to quasi-Banach spaces, Colloq. Math., to appear.

[18] H. Triebel, Modulation spaces on the Euclidean n-space, Z. Anal. Anwendungen 5 (1983), 443-457.

NuHAG, Faculty of Mathematics

University of Vienna

Nordbergstrasse 15

A-1090 Wien, Austria

E-mail:rauhut@univie.ac.at

Received July 5, 2005

Revised version April 28, 2007

(5693) 